\title{
Expansion Characteristics of a Plasma Jet in the Stepped-Wall Chamber Filled with Water
}

\author{
Yi Liu1, Yonggang Yu¹, Shanshan Mang² \\ ${ }^{1}$ School of Energy and Power Engineering, Nanjing University of Science and Technology, Nanjing, China \\ ${ }^{2}$ School of Science, Nanjing University of Science and Technology, Nanjing, China \\ Email: liuyi61mm@163.com
}

How to cite this paper: Liu, Y., Yu, Y.G. and Mang, S.S. (2019) Expansion Characteristics of a Plasma Jet in the Stepped-Wall Chamber Filled with Water. World Journal of Engineering and Technology, 7, 293-301. https://doi.org/10.4236/wjet.2019.72020

Received: March 27, 2019

Accepted: April 8, 2019

Published: April 15, 2019

\begin{abstract}
The interaction mechanism between the plasma and liquid is a key problem for the electrothermal chemical launch technology. To investigate this problem, a simulated experiment for the expansion process of a plasma jet in the working fluid is carried on. Based on this experiment, a two-dimensional axisymmetric unsteady theoretical model is established to reveal the plasma-liquid interaction flow field pattern. The results show that a typical Taylor cavity forms as the plasma jet expands in liquid. The induction effect of the stepped-wall structure enhances the radial expansion of the plasma jet. An arc-shaped pressure wave is generated at the front of the plasma jet and then evolves into the plane wave. A high-pressure area forms at the head of the plasma jet and then moves downstream. There is a strong plasma-liquid turbulent mixing at the interface, especially near the steps and the nozzle exit area.
\end{abstract}

\section{Keywords}

Electrothermal Chemical Launch, Plasma Jet, Stepped-Wall Chamber, Plasma-Liquid Interaction

\section{Introduction}

Electrothermal chemical launch technology, which is an advanced high-velocity propulsion technology, is widely interested by scholars [1]. The propulsive energy of electrothermal chemical gun consists of electrical energy input and the chemical energy from propellant combustion. Since the 1980s, more than a dozen countries such as the United States, Germany, and South Korea have started working on electrothermal chemical launch technology [2] [3] [4].

Electrothermal chemical guns can be classified into solid propellant electro- 
thermal chemical guns [5] [6] and liquid propellant electrothermal chemical guns (LPETCG). Compared with solid propellant, liquid propellant has a higher packing density and chemical energy and is easy to realize loading automation. Therefore, liquid propellant has a good development prospect. However, there are complex phenomena including heat transfer, plasma expansion, liquid breakup, droplet entrainment, and vortex evolution during the interior ballistics process of LPETC guns. There even is the gun bore explosion accident in the internal ballistic process of the LPETCG. The crucial reason is the core scientific problem - the interaction mechanism of the plasma jet and liquid is not clear now. Therefore, it is necessary to investigate the expansion process and characteristics of the plasma jet in liquid and find a suitable method to control the stability of the liquid propellant combustion.

The concept of using a stepped-wall structure in the liquid chamber was introduced as a potential mean of controlling the combustion of bulk-loaded liquid propellant [7], and this approach was further developed and tested by experimental and numerical research [8] [9]. Further, the stepped-wall structure in the liquid chamber is also an efficient way to control the plasma-liquid interaction. $\mathrm{Yu}$ [10] revealed that the axial expansion speed of the plasma jet in the stepped-wall liquid chamber is greater than in the traditional cylinder chamber. Zhang [11] found that the radial turbulence of the plasma jet is enhanced in the stepped-wall liquid chamber. Further, increasing the stepped-wall liquid chamber's extension factor (ratio of the step diameter to the step height) can reduce the axial turbulence of plasma jet [12].

In this work, the evolution process of a plasma jet in the stepped-wall liquid chamber with four steps was observed by the experiment. Based on the experiment, a two-dimensional axisymmetric unsteady mathematical physics model of a plasma jet expanding in water was established to study the expansion characteristics of a plasma jet, as well as the pressure evolution in the plasma-liquid interaction flow field. The results can provide a reference to investigate the interaction mechanism of the plasma jet and the ambient liquid.

\section{Experiment}

\subsection{Experimental Device}

To study the interaction mechanism of the plasma jet and the working fluid, an experimental device was designed including a pulse power source, a plasma generator, and liquid chamber. The pulse power source mainly included a pulse-forming network, as shown in Figure 1. The role of the plasma generator was to generate the high-temperature and high-pressure plasma. The plasma generator was the load of the pulse-forming network, so the plasma generation process could be controlled by setting discharge voltages.

The plasma generator (shown in Figure 2) mainly consists of polyethylene capillary, exploding wires, electrodes, insulators, and metal shells. The polyethylene capillary was connected to the pulse power source by a pair of electrodes. 


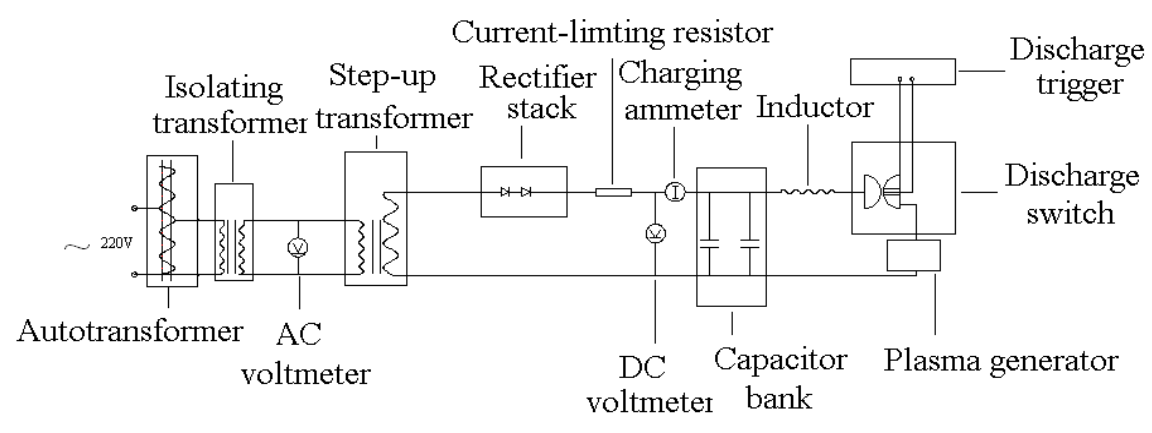

Figure 1. Pulse-forming network.
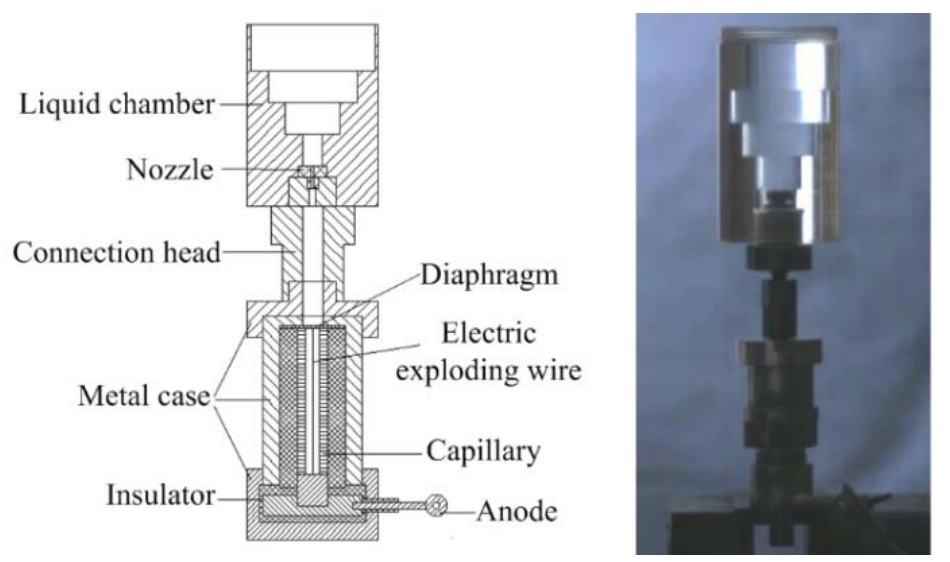

Figure 2. Plasma generator and liquid chamber.

The anode was sealed and the cathode was connected to the nozzle bottom of liquid chamber. This liquid chamber has four steps and the size details are shown in Table 1. Considering the experimental safety, water, which has a similar density and viscosity with the liquid propellant, was adopted as the liquid working medium in this experiment.

\subsection{Experimental Result}

During the experiment process, the pulse power source loaded electric energy between the anode and cathode of the plasma generator. The exploding wire in the plasma generator exploded, and the high-temperature metal plasma was generated. Then the metal plasma ablated the polyethylene on the capillary wall, and the plasma mixture is generated. When the pressure in the nozzle was high enough to break the copper film, the plasma jet was injected into the stepped-wall liquid chamber. The expansion process of a plasma jet in water, shown as Figure 3, was recorded by a FANSTCAM-ultima APX high-speed digital photographic system with 3000 frames per second.

A plasma cavity, called Taylor cavity, forms within the working fluid inside the liquid chamber. This cavity's axial expansion goes faster than its radial expansion as the plasma cavity expands along the step structure in the liquid chamber. These images show that the cavity boundary is blurred, especially at the later stage of the expansion process, because there is strong turbulent mixing 
Table 1. Structure size of the liquid chamber.

\begin{tabular}{cccc}
\hline Structure & Length/mm & Diameter $/ \mathrm{mm}$ & Diameter Increment $/ \mathrm{mm}$ \\
\hline step1 & 20 & 18 & $/$ \\
step2 & 20 & 30 & 12 \\
step3 & 20 & 42 & 12 \\
step4 & 38 & 54 & 12 \\
\hline
\end{tabular}

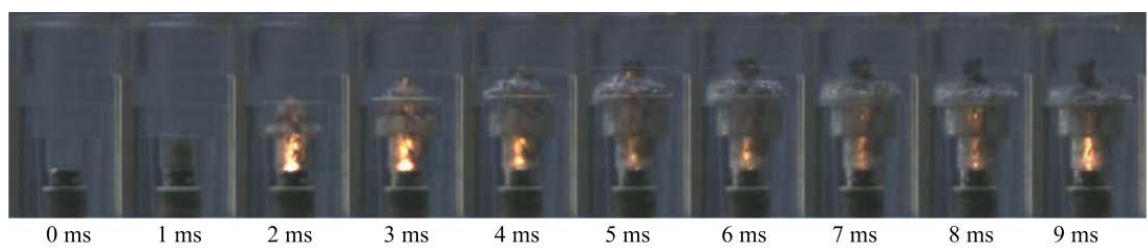

Figure 3. The expansion process of a plasma jet in the water.

of plasma and water at the interface. Further, this turbulence is stronger near the step corner than other areas, because the stepped-wall structure causes the reflow liquid which enhancing the plasma-liquid interaction at the step corner. In addition, the plasma cavity is brighter during the period of $2-4$ milliseconds, indicating the thermal energy of the plasma jet is greater at this period. Certainly, the shading effect caused by many bubbles and vapors should be not ignored in these graphs.

\section{Numerical Calculation}

\subsection{Theoretical Model}

An unsteady 2D theoretical model was established to simulate the expansion process of a plasma jet in liquid. Some physical assumptions are made as follows:

1) The Volume of Fluid model calculates the multiphase flow and the Standard k- $\varepsilon$ model simulates the plasma-liquid turbulent mixing.

2) The plasma-liquid interaction process studied in this work is short (not more than 10 milliseconds), so the liquid vaporization is ignored. The effect of the plasma sheath, as well as the effect of gravity, is also ignored to simplify the calculation.

3) The plasma jet is approximated as a high-temperature and high-pressure compressible fluid.

Based on the assumptions, governing equations are described as following:

1) Equation of continuity:

$$
\frac{\partial\left(\alpha_{q}\right)}{\partial t}+\boldsymbol{u} \cdot \nabla \alpha_{q}=0
$$

where $u$ is velocity, $\alpha_{q}$ is the volume fraction of $q$ th phase. The volume fraction of the primary phase (plasma), $\alpha_{1}$, will be calculated based on the constraint: $\alpha_{1}+\alpha_{2}+\alpha_{3}=1$.

2) Momentum equation: 


$$
\frac{\partial(\rho \boldsymbol{u})}{\partial t}+\nabla \cdot(\rho \boldsymbol{u} \boldsymbol{u})=-\nabla p+\nabla \cdot\left[\mu\left(\nabla \boldsymbol{u}+\nabla \boldsymbol{u}^{\mathrm{T}}\right)\right]
$$

where $\rho$ is density, $p$ is static pressure and $\mu$ is dynamic viscosity. $\boldsymbol{u}^{\mathrm{T}}$ is the transposed matrix of $\boldsymbol{u}$.

3) Energy equation:

$$
\frac{\partial(\rho E)}{\partial t}+\nabla \cdot(\boldsymbol{u}(\rho E+p))=\nabla \cdot\left(\mathrm{k}_{\mathrm{eff}} \nabla T\right)+S_{\mathrm{h}}
$$

where $\mathrm{k}_{\mathrm{eff}}$ is effective thermal conductivity, $E$ is energy and $T$ is temperature. The radiant energy $S_{\mathrm{h}}$ is given by

$$
S_{\mathrm{h}}=f \sigma\left(T-T_{0}\right)^{4}
$$

where $f$ is emissivity, $\sigma$ is Stefan-Boltzmann constant and $T_{0}$ is environment temperature.

4) State equation:

$$
p=\rho T \mathrm{R}
$$

where $\mathrm{R}$ is mixing gas constant.

5) Turbulence equation:

$$
\begin{gathered}
\frac{\partial}{\partial t}(\rho k)+\frac{\partial}{\partial x}\left(\rho k u_{x}\right)=\frac{\partial}{\partial r}\left[\left(\mu+\frac{\mu_{\mathrm{t}}}{\sigma_{\mathrm{k}}}\right) \frac{\partial k}{\partial r}\right]+G_{\mathrm{k}}+G_{\mathrm{b}}-\rho \varepsilon-Y_{\mathrm{M}} \\
\frac{\partial}{\partial t}(\rho \varepsilon)+\frac{\partial}{\partial x}\left(\rho \varepsilon u_{x}\right)=\frac{\partial}{\partial r}\left[\left(\mu+\frac{\mu_{\mathrm{t}}}{\sigma_{\varepsilon}}\right) \frac{\partial \varepsilon}{\partial r}\right]+C_{1 \varepsilon} \frac{\varepsilon}{k}\left(G_{\mathrm{k}}+C_{3 \varepsilon} G_{\mathrm{b}}\right)-C_{2 \varepsilon} \rho \frac{\varepsilon^{2}}{k}
\end{gathered}
$$

where $k$ is the turbulence kinetic energy, and $\varepsilon$ is its dissipation rate, $\sigma_{\mathrm{k}}$ and $\sigma_{\varepsilon}$ are the turbulent Prandtl numbers for $k$ and $\varepsilon$. In the equation, $\mu_{\mathrm{t}}=0.09 \rho k^{2} / \varepsilon$, $C_{1 \varepsilon}, C_{2 \varepsilon}$, and $C_{3 \varepsilon}$ are constants, $x$ is axial displacement, $r$ is radial displacement, $u_{x}$ is axial velocity. $G_{\mathrm{k}}$ represents the generation of turbulence kinetic energy due to the mean velocity gradients, $G_{\mathrm{b}}$ is the generation of turbulence kinetic energy due to buoyancy, $Y_{\mathrm{M}}$ represents the contribution of the fluctuating dilatation during the compressible turbulence to the overall dissipation rate.

\subsection{Calculation Domain and Mesh}

The axial symmetry of the theoretical model enables the computational domain adopted half of the plasma-liquid interaction flow field. The computational domain, as shown in Figure 4, includes nozzle, liquid chamber, and atmospheric

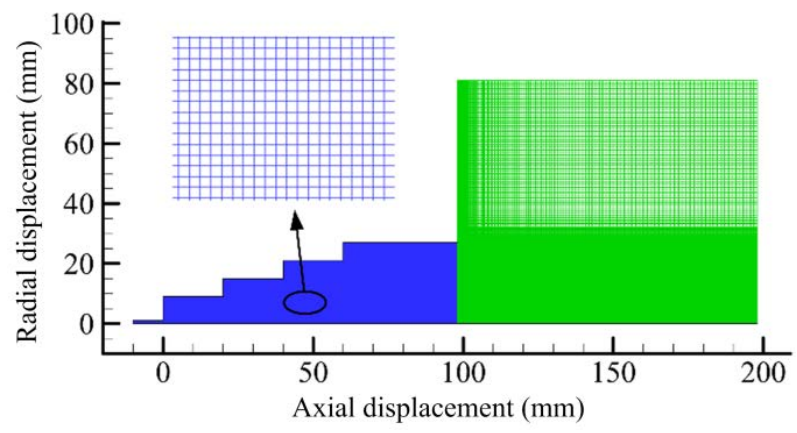

Figure 4. Calculation domain and mesh. 

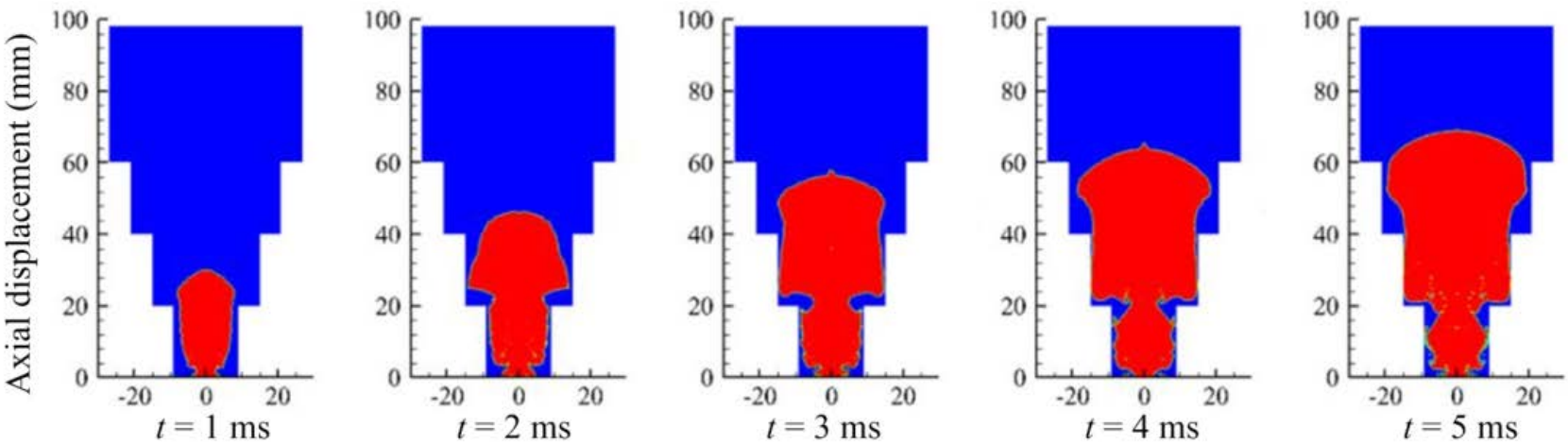

Radial displacement ( $\mathrm{mm}$ )

Figure 5. Phase distribution of the plasma-water interaction flow field. 
shown in Figure 6. Those curves are in a good agreement, indicating the calculation model is reasonable in this work.

The pressure distribution of plasma-liquid interaction flow field is shown in Figure 7. With the plasma-liquid interaction, an arc-shaped pressure wave is generated at the front of the interface and then evolves into the plane wave by the reflection of the chamber wall. A high-pressure area forms at the head of the plasma jet, because the plasma jet has a great compression at the jet head area. This high-pressure area moves downstream and gradually decreases with the plasma expansion in liquid. When this high-pressure area moves near the stepped-wall structure, it expands towards the chamber wall due to the radial induction effect of the steps. At the later expansion stage, this high-pressure area disappears because there is a pressure balance between water and plasma jet at this time. Besides, there is a local low-pressure area at the corner of the step due to the influence of stepped-wall structure in the liquid chamber.

\section{Conclusions}

The evolution process of a plasma jet in the stepped-wall liquid chamber with four steps was observed by the experiment. Based on the experiment, a two-dimensional axisymmetric unsteady mathematical physics model of a plasma jet expanding in water was established to study the plasma-liquid interaction mechanism. The calculated results agree well with the experimental results. The conclusions are obtained as follows.

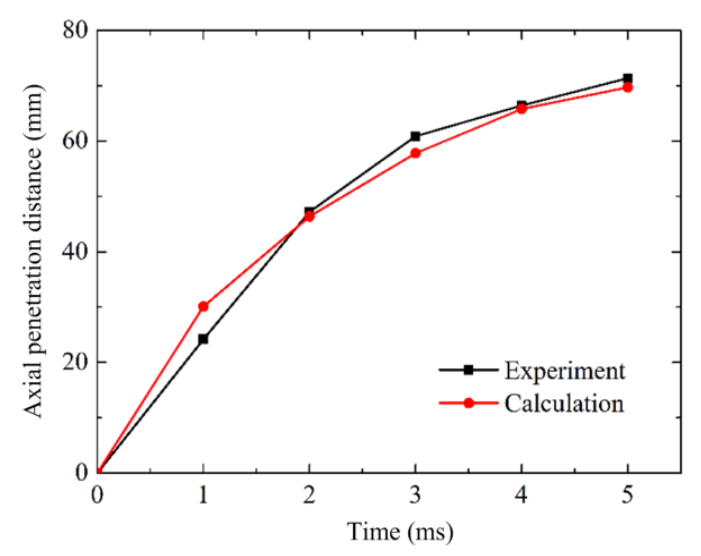

Figure 6. Axial penetration distance of plasma jet.
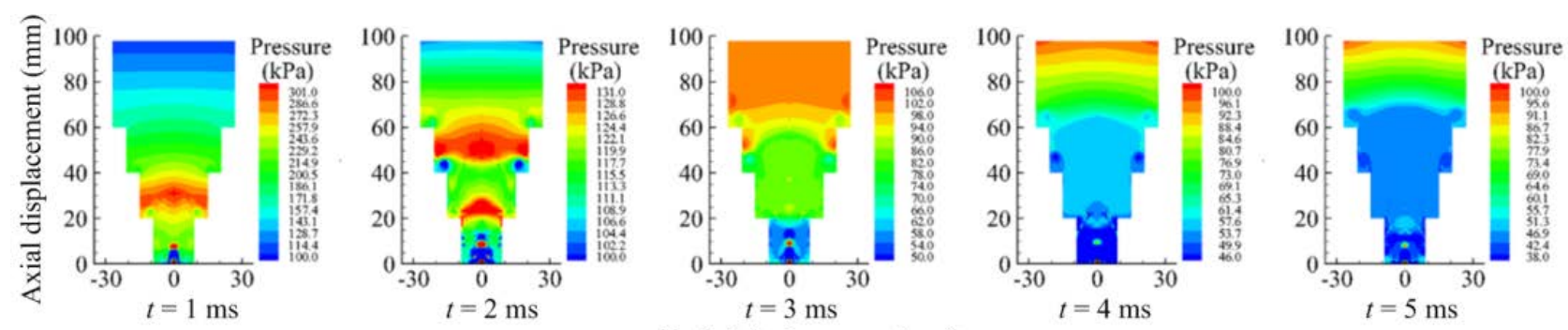

Radial displacement ( $\mathrm{mm}$ )

Figure 7. Pressure distribution of plasma-liquid interaction flow field. 
1) As the plasma jet expands in water, a plasma cavity, which forms within the water inside the liquid chamber, expands along the stepped wall. This cavity's axial expansion goes faster than its radial expansion. The radial induction effect of the stepped-wall structure enhances the radial expansion of the plasma jet. The plasma cavity is brighter during the period of 2 - 4 milliseconds in the sampling period. Besides, there is strong plasma-liquid turbulent mixing at the interface, especially near the steps and nozzle exit.

2) The plasma-liquid interaction has an effect on the pressure distribution in the flow field. An arc-shaped pressure wave is generated at the front of the plasma jet and then evolves into the plane wave. A high-pressure area forms at the head of the plasma jet and then moves downstream. Besides, there is a local low-pressure area at the corner of the step.

\section{Fund}

This work is greatly supported by the National Natural Science Foundation of China (Grant No. 51506094).

\section{Conflicts of Interest}

The authors declare no conflicts of interest regarding the publication of this paper.

\section{References}

[1] Roy, A.K., Lankennavar, P.H. and Ghadge, V.S. (2017) Present and Futuristic Trends in Weapon System. Defence Science Journal, 67, 401-405. https://doi.org/10.14429/dsj.67.11441

[2] Jung, J.W., Kim, S.H. and Yang, K.S. (2003) Overview of ETC Research in Korea. IEEE Transactions on Magnetics, 39, 22-23. https://doi.org/10.1109/TMAG.2002.805918

[3] Weise, T.H.G.G., Maag, J., Zimmermann, G., Eisenreich, N. and Derlich, H. (2003) National Overview of the German ETC Program. IEEE Transactions on Magnetics, 39, 35-38. https://doi.org/10.1109/TMAG.2002.805915

[4] Kim, S.H., Yang, K.S., Lee, Y.H., Kim, J.S. and Lee, B.H. (2009) ElectrothermalChemical Ignition Research on 120-mm Gun in Korea. IEEE Transactions on Magnetics, 45, 341-346. https://doi.org/10.1109/TMAG.2008.2008415

[5] Ni, Y.J., Jin, Y., Cheng, N.K., Yang, C.X., Li, H.Y. and Li, B.M. (2017) Simulation of Two-Dimensional Interior Ballistics Model of Solid Propellant ElectrothermalChemical Launch with Discharge Rod Plasma Generator. Defence of Thchnology, 13, 249-256. https://doi.org/10.1016/j.dt.2017.05.004

[6] Zhang, J.B., Li, X.W., Hang, Y.H. and Yang, W.H. (2018) An Electrothermal Plasma Model Considering Polyethylene and Copper Ablation Based on Ignition Experiment. Journal of Physics D: Applied Physics, 51, 1-15. https://doi.org/10.1088/1361-6463/aac0b0

[7] Talley, R.L. (1990) Diagnostics of Combustion Evolution in a Bulk-Loaded LP Gun. Proceedings of the 27 th Combustion Subcommittee Meeting, October 1990, 461-471.

[8] Xue, X.C., Yu, Y.G. and Mang, S.S. (2016) Physical Characteristics on High-Pressure Combustion and Propelling Process of Bulk-Loaded Energetic Liquid. Applied 
Thermal Engineer, 98, 1070-1079.

https://doi.org/10.1016/j.applthermaleng.2016.01.006

[9] Xue, X.C., Yu, Y.G. and Zhang, Q. (2014) Study on the Effect of Distance between the Two Nozzle Holes on Interaction of High Pressure Combustion-Gas Jets with Liquid. Energy Convers Manage, 85, 675-686.

https://doi.org/10.1016/j.enconman.2014.03.039

[10] Yu, Y.G., Yan, S.H., Zhao, N. and Zhang, Q. (2010) Influence of Boundary Shape on Interaction Process of Plasma Jet and Liquid Media. International Journal of Applied Electromagnetic Mechanism, 33, 541-548.

https://doi.org/10.3233/JAE-2010-1156

[11] Zhang, Q., Yu, Y.G., Lu, X. and Liu, D.Y. (2011) Experiment on Plasma-Liquid Interaction in a Stepped-Wall Chamber. Explos Shock Waves, 31, 311-316.

[12] Zhang, Q. and Yu, Y.G. (2013) Experimental Study on Boundary Shape Affecting Propagation Properties of Plasma Jet. Journal of Experimental Fluid Mechanism, 27, 15-19. 